\title{
Human.miRFFL.DB- A curated resource for human miRNA coregulatory networks and associated regulatory-circuits
}

\author{
Pankaj Khurana*, Rajeev Varshney, R Sugadev, YK Sharma, \\ Defence Institute of Physiology and Allied Sciences (DIPAS), Defence R\&D Organization \\ (DRDO), Timarpur, Delhi-110054
}

*Correspondence: pankajkhurana222@gmail.com ; Tel.: +91-11-23883116

\section{Highlights}

- microRNA (miRNA) and Transcriptional Factors (TFs) are two important regulatory biomolecules and a complex interplay between the two control a Target Gene (TG).

- The tripartite regulatory-circuits of TF, miRNA regulating a common TG is known as regulatory-circuit.

- Regulatory-circuits are extensively used to uncover underlying regulatory mechanisms in many physiological, pathophysiological and pathological conditions.

- Human.miRFFL.DB offers a comprehensive repository of 2,596 human miRNA:TF:TG coregulatory networks and associated regulatory circuits.

- Human.miRFFL.DB also provides an interactive visualization of coregulatory networks and the network motifs.

- The interplay between miRNAs, TFs and TGs help to get new mechanistic insights into complex molecular and cellular regulatory processes during complex multifactorial diseases.

\begin{abstract}
:
Gene regulation is viewed as a complex process where regulatory elements and their targets form highly complex network interactions thus affecting normal biological physiology and also disease-initiation and progress. Transcription factors (TF) and microRNA (miRNA) are fundamental transcriptional and post-transcriptional regulators of the gene expression controlling important biological processes. In recent years, many high throughput studies revealed that the complex regulatory interactions are mediated by the complex interplay between miRNA and TF regulating a Target Gene (TG) in conjunction. miRNAs and TFs are also known to regulate each other. This complex coregulatory mechanism may be represented in the form of miRNA:TF:TG coregulatory network. This network can be used to identify several small recurring subgraphs known as regulatory-circuits. One of these regulatory-circuits also called the Feed-Forward Loops (FFLs) is a three-node pattern which is composed of a
\end{abstract}


miRNA and a TF, one of which regulates the other and both jointly regulate a TG. These regulatory-circuits have proven useful in elucidating the complex interplay of gene regulation during many physiological and pathological conditions.

Human.miRFFL.DB is a comprehensive integrated resource for human miRNA:TF:TG coregulatory directed networks and their associated regulatory-circuits. In-house scripts based on the graph theory principle have been used to identify both types of FFL motifs i.e. miRNA-FFL and TF-FFL. The database additionally provides an interactive visualization of the coregulatory networks and associated FFLs. Human.miRFFL.DB can be used as a comprehensive ready reference for human miRNA:TF:TG coregulatory networks and associated FFLs for decrypting complex cellular interactions of these regulatory biomolecules. Human.miRFFL.DB is available online at http://mirffldb.in/human/

Keywords: miRNA:TF:TG coregulatory network, regulatory-circuits, miRNA-target interactions, Regulatory Networks

\section{Introduction}

Gene expression regulation is a complex process involving various regulatory biomolecules across numerous levels [1]. Transcription factors (TFs) and microRNAs (miRNAs) are the two most common regulatory biomolecules that fine-tune gene expression by regulating at transcriptional level and post-transcriptional level respectively [2]. They are known to regulate gene expression independently; but recent increasing evidence show that miRNAs and TFs also work synergistically in the form of complex networks to regulate the gene expression, which further modulates cellular and molecular processes [3]. Their combinatorial role in disease initiation, processes, and recurrence has also been studied [4-6]. These complex regulatory interactions can be best studied and analysed using miRNA:TF:TG co-regulatory networks. These co-regulatory networks are responsible for the impressive degree of complexity in gene-regulation in higher eukaryots [7]. Studies on recurrent circuits, also known as network motifs, in gene co-regulatory network have significantly contributed to uncover this complexity, and in better understanding the regulatory roles of miRNAs and TFs [7]. Specifically, miRNAs and TFs have been shown to regulate common genes in these regulatory-circuits [8]. One of these regulatory-circuits called Feed Forward Loops (FFLs) are tripartite motifs in which miRNA regulates TF and/or regulated by it, and both together regulate the Target Gene (TG) in the network [9]. Based on type of interaction between miRNA and TF, the FFLs can be broadly classified into 2 types: TF-FFL and miRNA-FFL[10]. In TF-FFL, the TF regulates the miRNA and the TG while the miRNA represses the same common TG. In miRNA-FFL, the miRNAis the master regulator as it represses both the TF and TG while the TF also regulates the same TG (Figure 1). At the network level, both miRNA-FFLs and TF-FFLs are important genetic overrepresented motif patterns that occur more often than by chance in biological networks. Hence they serve as basic building blocks of a complex regulatory system $[11,12]$. These FFLs are extensively studied to discover underlying genotypic and phenotypic relationship in complex disease conditions. e.g. TF: c-Myc; miR: miR-17-5p; target: E2F1 and 
c-Myc; miR: miR-20a; target: E2F1 were identified as module tightly regulating proliferative signal of proto-oncogene c- Myc and promoting cell cycle progression[13, 14]. C-Myc was termed as mater regulator of cellular proliferation in most of the human cancer types [15]. Hence c-Myc regulated FFL motifs in human cancer is extensively studied and a separate catalogue of C-myc regulating FFL motifs is consolidated [16]. Apart from the c-Myc other important FFLs regulating major molecular functions during cancer are TF: P53; miR: miR-34; target: MET, TF: E2F; miR: miR-106b/93/25; target: CDK, TF: AP-1; miR: miR-101; target: MMP9. These are known to regulate the extent of cancer invasion, anti-proliferative activity and cell migration respectively in different cancer studies [7]. A database of Cancer-Specific MicroRNA And Transcription Factor Co-Regulatory Networks (CMTCN) was recently developed to identify miRNA:TF:TG coregulatory network and associated FFLs in various human cancer types[17]. miRNA:TF:TG coregulatory networks and FFLs have also been found to be very useful in identifying mechanisms involved in other multifactorial disease for disease-initiation, -progression and -recurrence e.g. TF: MAX; miR: miR-320; target: BMP6 and TF: IRF1; miR: miR-103a-3p; target: ACVR2B was overrepresented in myocardial infarction recurrence condition and both targets BMP6, ACVR2B have been identified as biomarker for myocardial infarction recurrence[18]. Similarly TF: TP53; miR: mir-34b; target: CAV1, was demonstrated to be only dysregulated module in cardiac hypertrophy patients [19]. 9 FFLs, four miRNA-FFLs and five co-regulatory FFLs were found to be regulating cell-cycle regulation during a hypoxia stress[20].

There are many resources that catalogue the miRNA:TF:TG coregulatory network and associated FFLs e.g. RegNetwork: regulatory network repository for gene regulatory networks (GRNs) provides different types of regulatory interactions i.e'TF:TG', 'TF:miRNA', miRNA:TG' 'miRNA:TF' [21]. CircuitsDB: is a database that provides mixed miRNA/ TF-FFL circuits of 193 mature miRNAs and 130 pre-miRNAs of human and mouse[22]. TFmiR and MAGIA2 are webservers that uses miRNAs/mRNAs expression profile to identify significantly enriched TF and miRNAs interactions for construction of miRNA:TF:TG coregulatory network[23, 24]. Though there are several databases and tools available for FFL identification but these resources have certain limitations. Some database are not comprehensive, whereas others require an expression profile of miRNAs or TFs for identification of FFL. Hence, some FFLs which are biologically relevant may be lost during enrichment processes. Hence there is a vital need for a comprehensive global resource of human miRNA:TF:TG coregulatory networks and associated FFLs.

Here, we present Human.miRFFL.DB, a comprehensive user-friendly database of human miRNA: TF: TG coregulatory directed networks and their associated regulatory-circuits. The current version of Human.miRFFL.DB contains miRNA:TF:TG coregulatory networks of $\sim 2600$ human miRNAs. To construct miRNA:TF:TG coregulatory networks, Human.miRFFL.DB has an exhaustive list of 5,02,652 validated miRNA- Target Interactions (MTIs). The database stores experimentally validated MTIs with associated details like miRNA-target name, target Entrez ID, experimental validation type, and support experimental validation (Functional/Functionally Weak) and link to corresponding publication that are 
displayed in tabular format. Human.miRFFL.DB characterizes these MTIs as miRNA:TG or miRNA:TF interactions. TF:TG and TF:miRNA interactions are also added to construct miRNA:TF:TG directed coregulatory network. Human.miRFFL.DB offers interactive visualization of this network in which the nodes are color-coded for their easy characterization (TG as yellow, TF as green and miRNA as red). Human.miRFFL.DB uses in-house scripts based on graph theory principle to identify all tripartite miRNA-FFL and TF-FFL motifs from the network. It also provides an interactive visualization of each tripartite graph. The MTIs, miRNA-FFLs and TF-FFLs, can be downloaded in excel and PDF format. The network file can also be downloaded in .SIF format, which can be used with popular visualization software like Cytoscape, Gephi, BINA. Hence Human.miRFFL.DB can be considered as a comprehensive non-redundant catalogue of human miRNA:TF:TG coregulatory networks and its associated FFLs. We hope that Human.miRFFL.DB will catalyze research in understanding the complex crosstalk between miRNA, TF and TG. These motifs may offer mechanistic insights into the complex regulatory mechanisms. Combining it with experimental validation, these FFLs can identify novel players that can be used as diagnostic or prognostic markers and therapeutic targets for multifactorial complex disorders and pathophysiological conditions.

\section{Material and Methods}

\subsection{Data Collection and Processing}

To construct Human.miRFFL.DB database, a non-redundant list of human miRNA was retrieved from miRBase (version 22). To ensure uniformity in the nomenclature, the precursor miRNAs were mapped to their mature form using miRBase[25] and miRDB[26] repository. Dead/obsolete entries were removed. Experimentally validated target interactions of each human miRNA was fetched from public repositories i.e. mirTarBase[27] and miRecords[28] using multiMiR[29] R package. For each interaction, additional information like target name, target Entrez ID, support experiment details, support experimental validation (functional strong/functionally Weak) and the reference PMID was also fetched. Figure 1 schematically illustrates the general workflow for the collection of resource data for Human.miRFFL.DB.

\subsection{Data Enrichment}

The data was further enriched by labelling miRNA Target Intercations (MTIs) as miRNA: TG and miRNA:TF interaction by using the universal dataset of all human 3738 TFs and 
co-transcription factors (coTFs). A list of 3462, 3240, 1769, 1758, 3360, 1538 TFs were fetched from TFcheckpoint[30], DBD[31], ORFeome[32], TcoF-DB V2[33], TFCat[34], TFClass[35], TRANSFAC[36] databases respectively. A non-redundant list of 3292 unique TFs were obtained after removing duplicate entries. Further 958 coTFs were also identified from TcoF-DB V2 database [33]. Thus a combined list of 3798 unique TFs and coTFs was obtained. The TF: TG interactions were fetched from TRANSFAC, OregAnno 3.0 [37] and TRRUST V2[38] databases. TF:miRNA interactions were fetched from TransmiR[39] and PuTmiR[40] databases. Finally, all the dataset files were loaded as JavaScript Object Notation (JSON) files format and stored in the MongoDB database. Vis.js specifically was used to display miRNA: TF:TG networks.

\subsection{FFL Motif Identification.}

Each vertex in the network is labelled as $\mathrm{V}_{\mathrm{m}}, \mathrm{V}_{\mathrm{TF}}$ or $\mathrm{V}_{\mathrm{TG}}$. Where, $\mathrm{V}_{\mathrm{m}}$ refers to a miRNA, $\mathrm{V}_{\mathrm{TF}}$ refers to a TF and $V_{T G}$ refers to a TG. The edges are annotated as $E_{m t}, E_{m g}, E_{t g}, E_{t m}$ where $E_{m t}$ refers to edge from $V_{m}$ to $V_{T F}, E_{m g}$ refers to edge from $V_{m}$ to $V_{T G}, E_{t g}$ refers to edge from $V_{T F}$ to $V_{\text {gene }}$ and $E_{t m}$ refers to edge from $V_{T F}$ to $V_{m}$.

For each $\mathrm{Vm}$, its edges $\mathrm{E}_{\mathrm{mg}}$ from the miRNA:TG interactions is searched in miRNA:TF interaction to identify a corresponding Emt with a common vertex Vm. Thereafter, an analogous Etg is searched in the TF:TG interactions. If found, the complete graph containing three vertices $\left(\mathrm{Vm}, \mathrm{V}_{\mathrm{TF}}, \mathrm{V}_{\mathrm{TG}}\right)$ and edges $\left(\mathrm{E}_{\mathrm{mt}}, \mathrm{E}_{\mathrm{mg}}, \mathrm{E}_{\mathrm{tg}}\right)$ were labelled as miRNA-FFL motif graph

Similarly in order to find TF-FFL motif, edge $\mathrm{E}_{t m}$ and $E_{t g}$ were identified from TF-miRNA and miRNA:TG interactions dataset respectively. Further the program scans for an $\mathrm{E}_{\mathrm{mg}}$ associated with $\mathrm{V}_{\mathrm{m}}$ and $\mathrm{V}_{\mathrm{TG}}$ in miRNA:TG interaction dataset. If algorithm finds $\mathrm{E}_{\mathrm{mg}}$, the complete graph containing three vertices $\left(\mathrm{Vm}, \mathrm{V}_{\mathrm{TF}}, \mathrm{V}_{\mathrm{TG}}\right)$ and edges $\left(\mathrm{E}_{\mathrm{tm}}, \mathrm{E}_{\mathrm{mg}}, \mathrm{E}_{\mathrm{tg}}\right)$ were labelled as TF-FFL motif graph. This methodology was used to identify miRNA-FFLs and TF-FFLs for each human miRNA. 


\subsection{Database development}

All database files were collected, processed and stored as JavaScript Object Notation (JSON) files in MongoDB database[41]. MongoDB is an open-source document-oriented (NoSQL) database $[41,42]$. The variables and query functions of the application program interface (API) in the Human.miRFFL.DB web application are defined in the python language. The database uses Asynchronous JavaScript and XML (AJAX) technique for API calls[43]. AJAX is a web development technique that is used for creating interactive web applications. It utilizes XHTML for content along with the document object model and JavaScript for dynamic content display. Vis.js library functions are used for interactive visualization of network graphs on front-end.

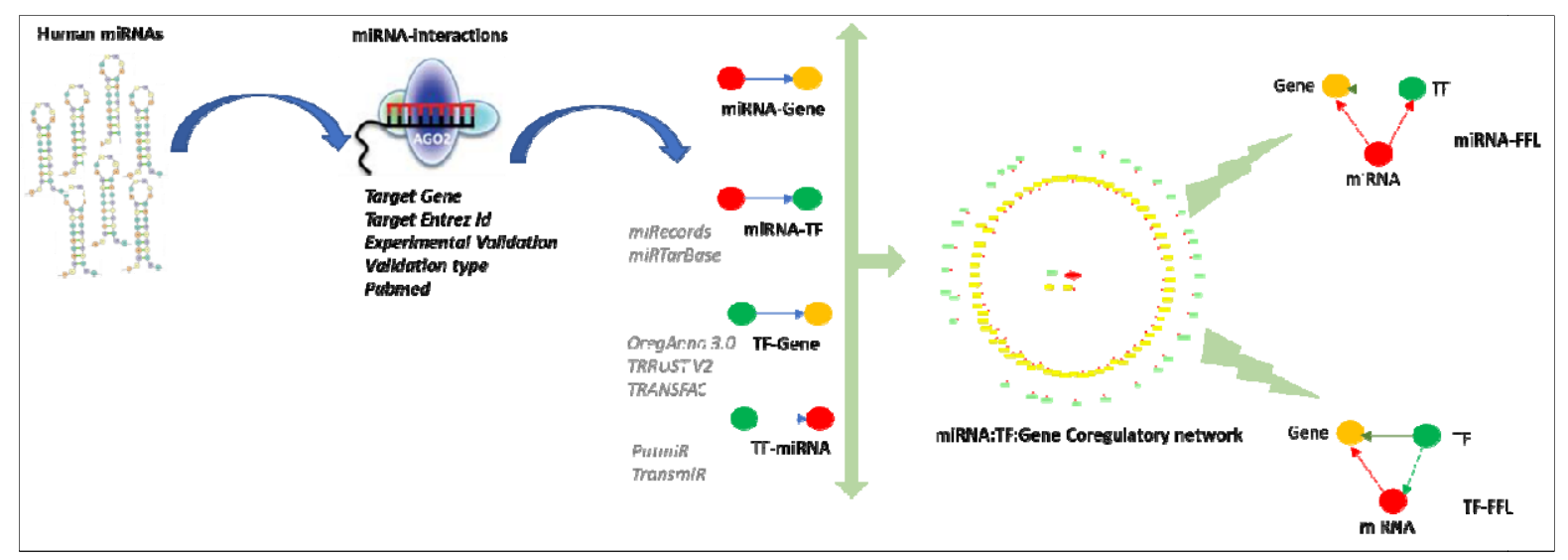

Figure 1: Overview of data collection and annotation in Human.miRFFL.DB database.

\section{RESULTS}

Human.miRFFL.DB is a comprehensive non-redundant resource for human miRNA:TF:TG coregulatory directed networks and their associated regulatory-circuits. It contains a non-redundant list of 2596 human miRNAs, 11,864 experimentally validated TGs and 3,798 TFs including co-Transcription cofactors (coTFs) (Figure 2a). The database contains $\sim 5,00,000$ experimentally validated MTIs mined from mirTarBase[27], miRecords[28] and 7200 corresponding reference PMIDs. The $\sim 3,80,000$ unique miRNA-target interactions are categorized as $\sim 1,14,000$ miRNA: TF interactions and $~ 2,66,000$ miRNA: TG interactions. 
Additionally, 49,000 TF:TG interactions were fetched from OregAnno[37] and TRRUST V2[38] databases and 1500 TF:miRNA interactions were fetched from TransmiR[39] and PuTmiR[40] (Figure 2b). These interactions were used to construct miRNA:TF:TG coregulatory directed network for each human miRNA.

a)

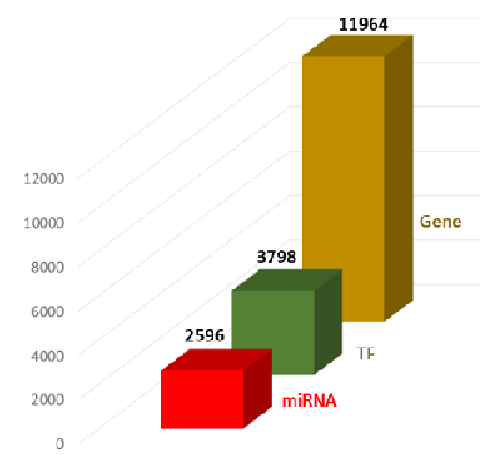

b)

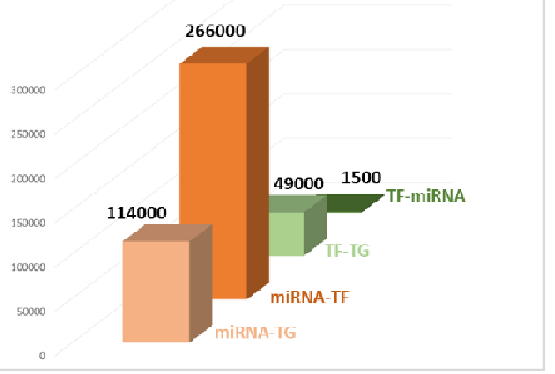

Figure 2 a) Number of miRNAs, TFs and TGs in Human.miRFFL.DB. b) Number of miRNA:TG, miRNA:TF, TF:TG, TF:miRNA interactions in the database.

\subsection{Web Interface}

Human.miRFFL.DB offers browsing by three routes i.e. 'search by miRNA', 'search by TF(s)' or 'search by gene(s)' (Figure 2). 'Search by miRNA' allows the user to select miRNA from a pull down menu. On clicking the search button, the page connects to the detailed information page of the respective miRNA, which is discussed in detail in next section 2.2. The database also facilitates the user to search the database by selecting $\mathrm{TF}(\mathrm{s})$ and $\mathrm{TG}(\mathrm{s})$ from a pull down menu. On searching the user can view the FFLs containing the queried TF(s) or gene(s) in a tabular format (Figure 2b, 2c). The table can be downloaded in Excel and PDF format. The miRNAs of the FFLs are further hyperlinked to the corresponding information page. 


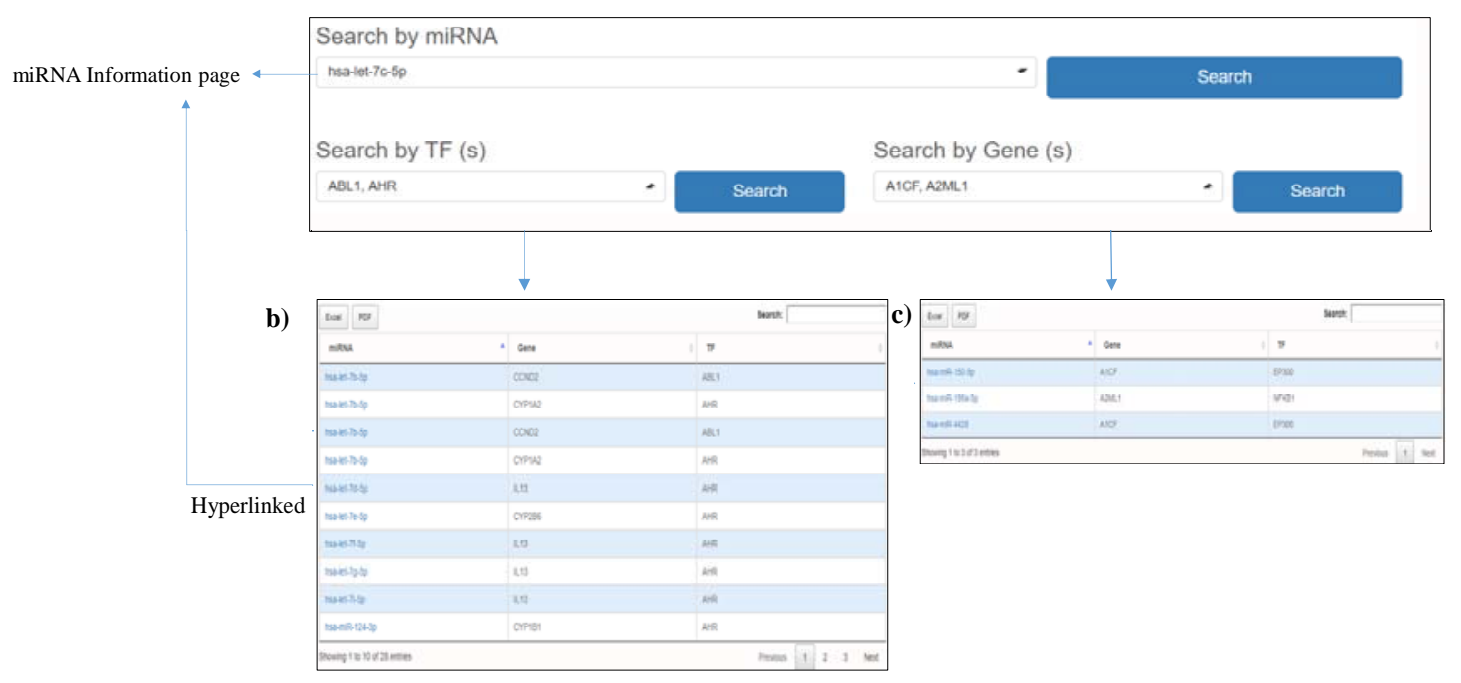

Figure 3: The figure shows the screenshot of the 'Browse' option. This option allow the users to browse miRNAs using three different options: 'Search by miRNA', 'Search by TF(s)' and 'Search by Gene(s)'.a) On searching with miRNA, the page connects to the detailed information page of the respective human miRNA. b) Table representing the FFLs containing the queried $\mathrm{TF}(\mathrm{s}) \mathrm{c}$ ) Table representing the FFLs containing the queried gene(s).

\section{2 miRNA Information Page}

The miRNA information page for each human miRNA can be divided into two sections. The first section displays interactive visualization of miRNA:TF:TG coregulatory network and a tabular representation of miRNA-FFLs and TF-FFLs that are identified from the network. The second section enlists the experimentally-validated MTIs and its associated information in a tabular format.

\subsection{1 miRNA:TF:TG co-regulatory network and FFLs}

The top of the miRNA information page highlights the name of the mature miRNA which is hyperlinked to miRBase database[25]. miRBase database is a comprehensive resource of miRNA sequence data, annotation and predicted gene targets. Hence hyperlinking each human miRNA in the database serve as a ready reference to get additional details about these miRNAs that includes mature and precursor miRNA sequence, miRNA stem loop structure, chromosome location, source organism etc (Figure 4). 
The miRNA:TF:TG network is displayed as an interactive network in which the nodes are color coded for easy characterization (miRNA in red and its interacting TFs and TGs in green and yellow respectively) (Figure 4a). The network is also equipped with zoom-in, zoom-out, and node-translation options. The network file can be downloaded in (.SIF) format and can be readily used by many open-source software's such as Cytoscape[44], Gephi[45], BINA[46], etc for further analysis.

The miRNA-FFL and TF-FFL are identified from the coregulatory network using in-house scripts and presented as separate tables. Each row of the table represents a FFL with a miRNA, TF and TG. User can also visualize tripartite graph by clicking on the miRNA, which will appear in a pop-up window (Figure 4a). Both the miRNA-FFL and TF- FFL tables are provided with an integrated search option. This allows the user to search the lengthy tables by TF/gene symbol. Each TF and TG symbol in the FFL table are further hyperlinked to the GeneCards[47] database. This would serve as a ready reference for the user to get additional details about the gene that includes aliases of genes, promoter and enhancer location of gene, protein coded by gene, functional characterization, cellular localization, pathway enrichment, gene-gene interaction network, drug-gene relationship, tissue specific gene expression profile, orthologs, paralogs, transcript variant etc.

\subsubsection{Experimentally validated MTIs}

Human.miRFFL.DB also provide experimentally validated MTIs along with its associated information that includes miRNA-target symbol, miRNA-target Entrez ID, experimental validation type, and support experimental validation (Non-Functional/Functional/Functionally Weak) and the reference paper. The information is provided in a tabular format that can be downloaded in the form of excel/PDF file. The table is also provided with an integrated search option, which allows to search the lengthy tables. The user may search with target symbol, entrez ID, experiment type, support type or PMID (Figure 4b). The PMID is hyperlinked to the PubMed database for ready access to the publication. 
a)
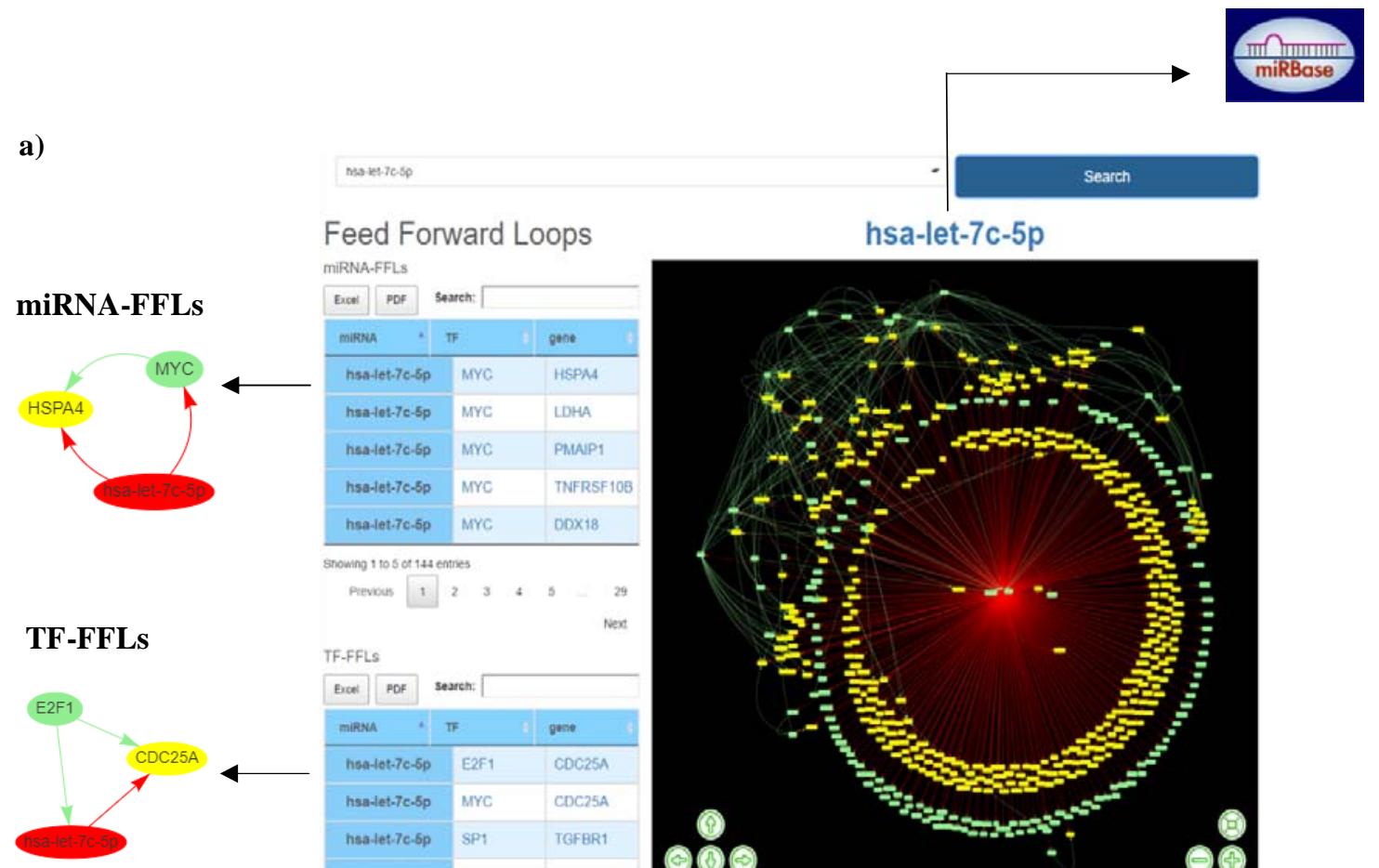

b)

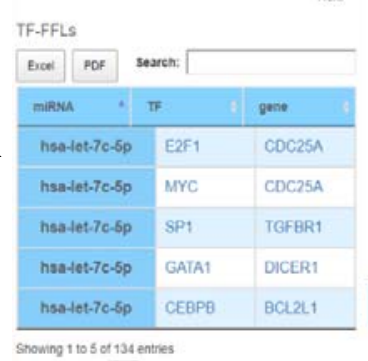

pieveus $12_{2} \quad 3 \quad 4 \quad 5$

Experimentally validated targets

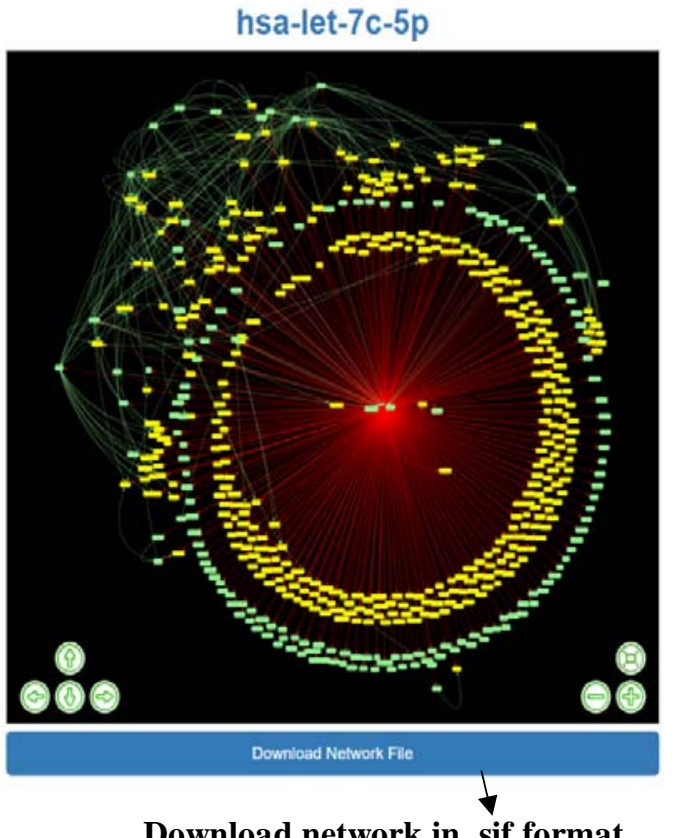

Download network in .sif format

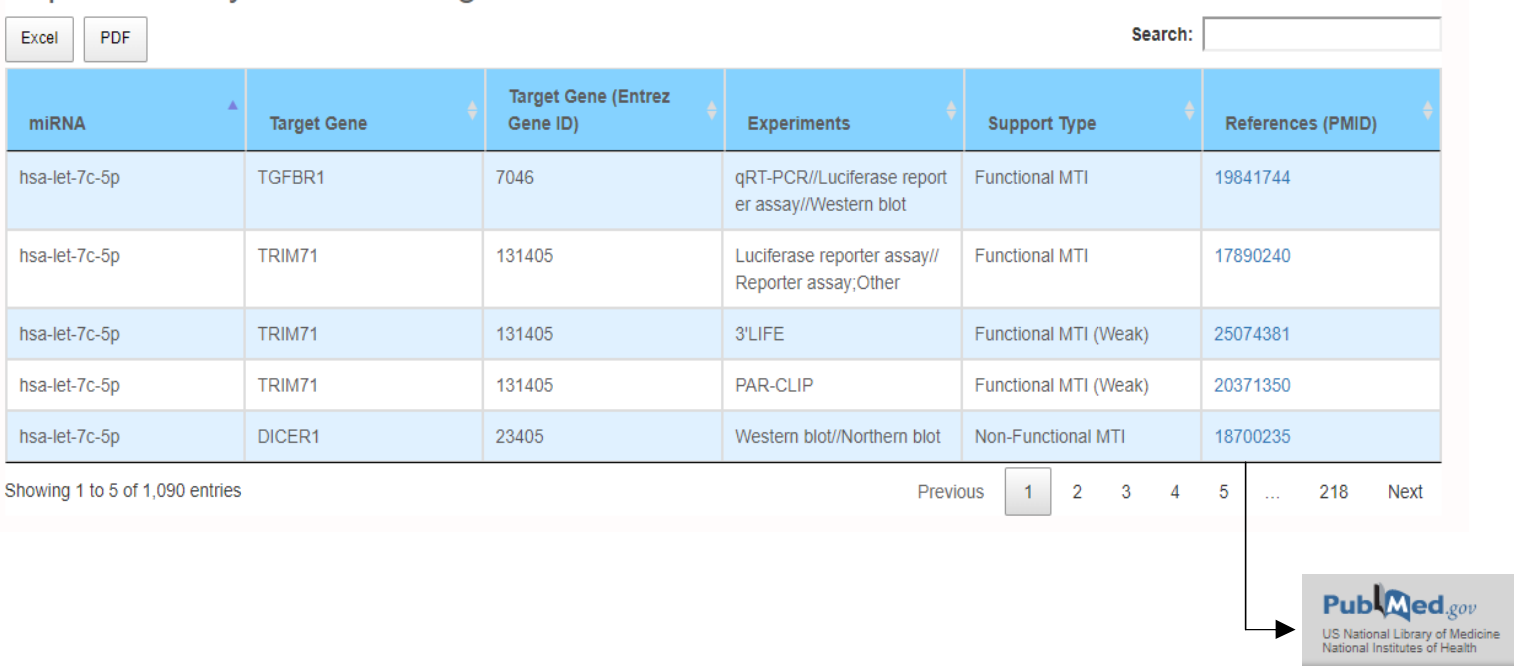

Figure 4: The web image of miRNA information page of hsa-let-7c-5p. a) The first section shows the coregulatory network and the associated miRNA-FFLs and TF-FFLs. Clicking on the miRNA, a pop-up window displays the corresponding FFL. The gene names are hyperlinked to GeneCards database, which provides additional details about the gene. b) The experimentally validated targets of the miRNA are listed along with the details like Target gene entrez ID, experiment, support type and the respective reference paper. The PMID is hyperlinked to the PubMed database for ready access to the publication. 


\section{Discussion}

A decade earlier, Yitzhak Pilpel et al studied the global miRNA:TF:TG mammalian co-regulatory network and uncovered two network architecture of regulatory network [48]. The network analysis of this global miRNA:TF mammalian co-regulatory network revealed several recurring motifs of miRNA and TF regulating a hub gene. These motifs or regulatory-circuits help in fine-tuning of many complex molecular and cellular processes. Since then, studies on recurrent circuits in regulatory networks, also known as network motifs, have substantially contributed to addressing this complexity and in particular to better understand miRNAs and TFs exert their regulatory roles[5, 7, 9]. The most studied recurring motifs in miRNA:TF:TG coregulatory networks are FFLs. FFLs have been proven to be the best network analysis tool to study the combinatorial TG regulation by miRNA and TF in many complex pathologies and are crucial in providing new insights into the logic and evolution of a new regulatory layer of the complex eukaryotic genome. Human.miRFFL.DB offers a comprehensive and interactive collection of human miRNA:TF:TG coregulatory networks and associated regulatory-circuits. Human.miRFFL.DB pools miRNA:TG, miRNA:TF, TF:TG and TF:miRNA interactions for constructing complex miRNA:TF:TG coregulatory networks of human miRNAs. These networks were used to identify the miRNA-FFL and TF-FFL motifs. Additionally, Human.miRFFL.DB also provides experimentally validated miRNA-Target Interactions (MTIs) resources for these human miRNAs.

A thorough literature survey suggested that though there are several server platforms, databases, and tools available for identification of gene regulatory networks (GRNs) and associated FFLs; all these research tools lack in addressing the core of the problem according to the current scenario e.g. CircuitsDB: a database for mixed miRNA/ TF FFL circuits in human and mouse contains information of only 193 mature miRNAs and 130 pre-miRNAs FFLs. Whereas, currently more than $\sim 2600$ human mature miRNAs (miRBase release 22) and 5,00,000 experimentally validated MTIs have already been identified[22, 27, 49]. RegNetwork: an integrated resource of transcriptional and post-transcriptional regulatory networks provides a 
collection of regulatory interactions among TFs, miRNAs and target genes. [21]. The database, however, does not provide FFLs. Also in the thorough examination of the database, many interactions were found missing. A random example of hsa-miR-141-3p has been used to compare the regulatory interactions present in our database (Table S1). Server tools such as TFmiR, MAGIA2 require miRNAs/mRNAs expression profile as input to identify regulatory interactions based on the input deregulated genes and deregulated miRNAs [23, 24]. For identification of regulatory interactions, they first identify miRNAs, whose target genes, as well as TFs, are significantly enriched within the input deregulated genes by using the hypergeometric distribution function. Hence only interactions among these miRNAs and significantly enriched TF, TGs are used to construct miRNA:TF:TG coregulatory network [23, 24]. This methodology reduces a significant amount of interactions from the network. Subsequently, it would also lead to the loss of important FFLs identified from the network. Databases like 'CMTCN' [17] and 'curated database of miRNA mediated feed-forward loops including MYC as master regulator'[16] provide only the cancer-specific miRNA:TF:TG coregulatory networks and associated FFLs. Though these databases are recently published their global coverage is limited to only cancer. FFLs have also been identified in many other multifactorial diseases and pathophysiological conditions like myocardial infarct, schizophrenia, and hypoxia respectively. So these databases, webservers, and tools cannot be considered universal repository for miRNA:TF:TG coregulatory networks and FFLs. Herein, we developed Human.miRFFL.DB which is a comprehensive coregulatory network resource that integrates miRNA:TG interactions, miRNA:TF interactions, TF:TG interactions and TF: miRNA interactions to build co-regulatory networks. These complex networks provide a global representation of complex regulatory and target interactions of human miRNAs and TFs. It can advance our understanding of complex molecular mechanisms that are controlled by these regulatory molecules.

\section{Conclusion}

Human.miRFFL.DB identifies FFLs that will help to decode the interplay between miRNAs, TFs and TGs to get new mechanistic-insights into specific molecular and cellular processes. 
Further analysis of these FFL motifs using network biology techniques could also help us in identifying potential disease-markers and therapeutic targets. The resource can also be used for other network-based computational and integrated analysis. This would allow researchers to be more focused on analysis rather than going for manual curation of the interactions from different databases to construct these co-regulatory networks. Also it is ready reference of enriched FFLs for human miRNA pool. We believe that Human.miRFFL.DB will help researchers to design and also analyze miRNA based experiments.

\section{Supplementary Materials:}

Table S1: Tabular representation of comparison in regulatory interactions of the hsa-miR-141-5p miRNA present in RegNetwork database with Human.miRFFL.DB.

\section{Declaration of competing interest-}

The authors declare that they have no known competing interests.

\section{Abbreviations}

$\begin{array}{ll}\text { TF } & \text { Transcription factor } \\ \text { TG } & \text { Target Gene } \\ \text { FFL } & \text { Feed Forward Loop } \\ \text { MTI } & \text { miRNA-Target Interaction } \\ \text { coTF } & \text { Co Transcription Factor }\end{array}$

\section{References}

[1] Macneil LT, Walhout AJ. Gene regulatory networks and the role of robustness and stochasticity in the control of gene expression. Genome research. 2011;21:645-57.

[2] Martinez NJ, Walhout AJ. The interplay between transcription factors and microRNAs in genome-scale regulatory networks. BioEssays : news and reviews in molecular, cellular and developmental biology. 2009;31:435-45.

[3] Feng Z, Zhang W, Xu J, Gauron C, Ducos B, Vriz S, et al. Optical control and study of biological processes at the single-cell level in a live organism. Reports on progress in physics Physical Society. 2013;76:072601.

[4] Jiang W, Mitra R, Lin CC, Wang Q, Cheng F, Zhao Z. Systematic dissection of dysregulated transcription factor-miRNA feed-forward loops across tumor types. Briefings in bioinformatics. 2016;17:996-1008.

[5] Xu Y, Yue W, Yao Shugart Y, Li S, Cai L, Li Q, et al. Exploring Transcription Factors-microRNAs Co-regulation Networks in Schizophrenia. Schizophrenia bulletin. 2016;42:1037-45. 
[6] Lin Y, Sibanda VL, Zhang HM, Hu H, Liu H, Guo AY. MiRNA and TF co-regulatory network analysis for the pathology and recurrence of myocardial infarction. Scientific reports. 2015;5:9653.

[7] Cora D, Re A, Caselle M, Bussolino F. MicroRNA-mediated regulatory circuits: outlook and perspectives. Physical biology. 2017;14:045001.

[8] Yan Y, Shi Y, Wang C, Guo P, Wang J, Zhang CY, et al. Influence of a high-altitude hypoxic environment on human plasma microRNA profiles. Scientific reports. 2015;5:15156. [9] Zhao Q, Liu H, Yao C, Shuai J, Sun X. Effect of Dynamic Interaction between microRNA and Transcription Factor on Gene Expression. BioMed research international.

2016;2016:2676282.

[10] Li R, Jiang S, Li W, Hong H, Zhao C, Huang X, et al. Exploration of prognosis-related microRNA and transcription factor co-regulatory networks across cancer types. RNA biology. 2019;16:1010-21.

[11] Anastasiadou E, Jacob LS, Slack FJ. Non-coding RNA networks in cancer. Nature reviews Cancer. 2018;18:5-18.

[12] Guo Y, Alexander K, Clark AG, Grimson A, Yu H. Integrated network analysis reveals distinct regulatory roles of transcription factors and microRNAs. Rna. 2016;22:1663-72.

[13] Pickering MT, Stadler BM, Kowalik TF. miR-17 and miR-20a temper an E2F1-induced G1 checkpoint to regulate cell cycle progression. Oncogene. 2009;28:140-5.

[14] Wu Q, Qin H, Zhao Q, He XX. Emerging role of transcription factor-microRNA-target gene feed-forward loops in cancer. Biomedical reports. 2015;3:611-6.

[15] Miller DM, Thomas SD, Islam A, Muench D, Sedoris K. c-Myc and cancer metabolism. Clinical cancer research : an official journal of the American Association for Cancer Research. 2012;18:5546-53.

[16] El Baroudi M, Cora D, Bosia C, Osella M, Caselle M. A curated database of miRNA mediated feed-forward loops involving MYC as master regulator. PloS one. 2011;6:e14742.

[17] Li R, Chen H, Jiang S, Li W, Li H, Zhang Z, et al. CMTCN: a web tool for investigating cancer-specific microRNA and transcription factor co-regulatory networks. PeerJ.

2018;6:e5951.

[18] Zhang G, Shi H, Wang L, Zhou M, Wang Z, Liu X, et al. MicroRNA and transcription factor mediated regulatory network analysis reveals critical regulators and regulatory modules in myocardial infarction. PloS one. 2015;10:e0135339.

[19] Qu W, Shi S, Sun L, Zhang F, Zhang S, Mu S, et al. Construction of a microRNAassociated feedforward loop network that identifies regulators of cardiac hypertrophy and acute myocardial infarction. International journal of molecular medicine. 2018;42:2062-70.

[20] Gupta A, Ragumani S, Sharma YK, Ahmad Y, Khurana P. Analysis of Hypoxiamir-Gene Regulatory Network Identifies Critical MiRNAs Influencing Cell-Cycle Regulation Under Hypoxic Conditions. MicroRNA. 2019;8:223-36.

[21] Liu ZP, Wu C, Miao H, Wu H. RegNetwork: an integrated database of transcriptional and post-transcriptional regulatory networks in human and mouse. Database : the journal of biological databases and curation. 2015;2015. 
[22] Friard O, Re A, Taverna D, De Bortoli M, Cora D. CircuitsDB: a database of mixed microRNA/transcription factor feed-forward regulatory circuits in human and mouse. BMC bioinformatics. 2010;11:435.

[23] Hamed M, Spaniol C, Nazarieh M, Helms V. TFmiR: a web server for constructing and analyzing disease-specific transcription factor and miRNA co-regulatory networks. Nucleic acids research. 2015;43:W283-8.

[24] Bisognin A, Sales G, Coppe A, Bortoluzzi S, Romualdi C. MAGIA(2): from miRNA and genes expression data integrative analysis to microRNA-transcription factor mixed regulatory circuits (2012 update). Nucleic acids research. 2012;40:W13-21.

[25] Griffiths-Jones S, Grocock RJ, van Dongen S, Bateman A, Enright AJ. miRBase: microRNA sequences, targets and gene nomenclature. Nucleic acids research. 2006;34:D140-4. [26] Wong N, Wang X. miRDB: an online resource for microRNA target prediction and functional annotations. Nucleic acids research. 2015;43:D146-52.

[27] Hsu SD, Tseng YT, Shrestha S, Lin YL, Khaleel A, Chou CH, et al. miRTarBase update 2014: an information resource for experimentally validated miRNA-target interactions. Nucleic acids research. 2014;42:D78-85.

[28] Xiao F, Zuo Z, Cai G, Kang S, Gao X, Li T. miRecords: an integrated resource for microRNA-target interactions. Nucleic acids research. 2009;37:D105-10.

[29] Ru Y, Kechris KJ, Tabakoff B, Hoffman P, Radcliffe RA, Bowler R, et al. The multiMiR R package and database: integration of microRNA-target interactions along with their disease and drug associations. Nucleic acids research. 2014;42:e133.

[30] Chawla K, Tripathi S, Thommesen L, Laegreid A, Kuiper M. TFcheckpoint: a curated compendium of specific DNA-binding RNA polymerase II transcription factors.

Bioinformatics. 2013;29:2519-20.

[31] Kummerfeld SK, Teichmann SA. DBD: a transcription factor prediction database. Nucleic acids research. 2006;34:D74-81.

[32] Lamesch P, Li N, Milstein S, Fan C, Hao T, Szabo G, et al. hORFeome v3.1: a resource of human open reading frames representing over 10,000 human genes. Genomics.

2007;89:307-15.

[33] Schmeier S, Alam T, Essack M, Bajic VB. TcoF-DB v2: update of the database of human and mouse transcription co-factors and transcription factor interactions. Nucleic acids research. 2017;45:D145-D50.

[34] Fulton DL, Sundararajan S, Badis G, Hughes TR, Wasserman WW, Roach JC, et al. TFCat: the curated catalog of mouse and human transcription factors. Genome biology. 2009;10:R29.

[35] Wingender E, Schoeps T, Haubrock M, Donitz J. TFClass: a classification of human transcription factors and their rodent orthologs. Nucleic acids research. 2015;43:D97-102. [36] Matys V, Fricke E, Geffers R, Gossling E, Haubrock M, Hehl R, et al. TRANSFAC: transcriptional regulation, from patterns to profiles. Nucleic acids research. 2003;31:374-8. [37] Lesurf R, Cotto KC, Wang G, Griffith M, Kasaian K, Jones SJ, et al. ORegAnno 3.0: a community-driven resource for curated regulatory annotation. Nucleic acids research. 2016;44:D126-32. 
[38] Khan A, Fornes O, Stigliani A, Gheorghe M, Castro-Mondragon JA, van der Lee R, et al. JASPAR 2018: update of the open-access database of transcription factor binding profiles and its web framework. Nucleic acids research. 2018;46:D1284.

[39] Tong Z, Cui Q, Wang J, Zhou Y. TransmiR v2.0: an updated transcription factor-microRNA regulation database. Nucleic acids research. 2019;47:D253-D8.

[40] Bandyopadhyay S, Bhattacharyya M. PuTmiR: a database for extracting neighboring transcription factors of human microRNAs. BMC bioinformatics. 2010;11:190.

[41] Schulz WL, Nelson BG, Felker DK, Durant TJS, Torres R. Evaluation of relational and NoSQL database architectures to manage genomic annotations. Journal of biomedical informatics. 2016;64:288-95.

[42] Liu J, Qu Z, Yang M, Sun J, Su S, Zhang L. Jointly integrating VCF-based variants and OWL-based biomedical ontologies in MongoDB. IEEE/ACM transactions on computational biology and bioinformatics. 2019.

[43] Berger SI, Iyengar R, Ma'ayan A. AVIS: AJAX viewer of interactive signaling networks. Bioinformatics. 2007;23:2803-5.

[44] Shannon P, Markiel A, Ozier O, Baliga NS, Wang JT, Ramage D, et al. Cytoscape: a software environment for integrated models of biomolecular interaction networks. Genome research. 2003;13:2498-504.

[45] Jacomy M, Venturini T, Heymann S, Bastian M. ForceAtlas2, a continuous graph layout algorithm for handy network visualization designed for the Gephi software. PloS one.

2014;9:e98679.

[46] Gerasch A, Faber D, Kuntzer J, Niermann P, Kohlbacher O, Lenhof HP, et al. BiNA: a visual analytics tool for biological network data. PloS one. 2014;9:e87397. [47] Safran M, Dalah I, Alexander J, Rosen N, Iny Stein T, Shmoish M, et al. GeneCards Version 3: the human gene integrator. Database : the journal of biological databases and curation. 2010;2010:baq020.

[48] Shalgi R, Lieber D, Oren M, Pilpel Y. Global and local architecture of the mammalian microRNA-transcription factor regulatory network. PLoS computational biology. 2007;3:e131. [49] Kozomara A, Birgaoanu M, Griffiths-Jones S. miRBase: from microRNA sequences to function. Nucleic acids research. 2019;47:D155-D62. 


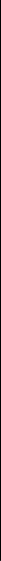


a)

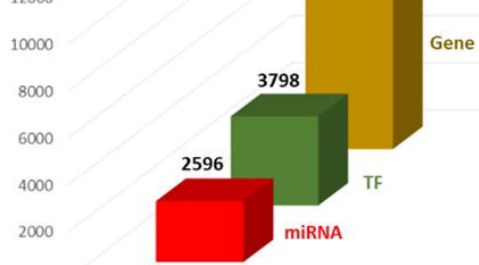

b)

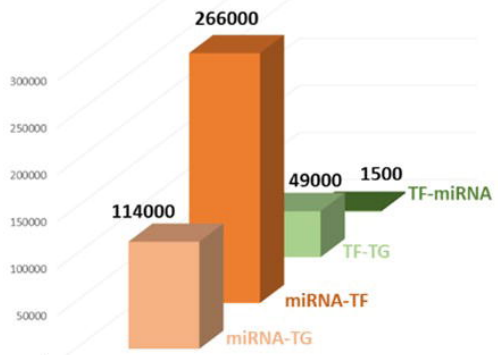


Search by miRNA

miRNA Information page

hsa-let-7c-5p

$-$

Search

Search by TF (s)

ABL1, AHR

4

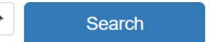

Search by Gene (s)

A1CF, A2ML

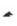

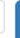

Search

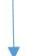

b)

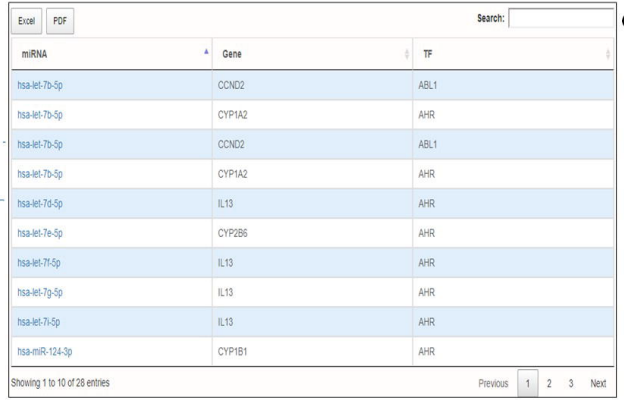

c)

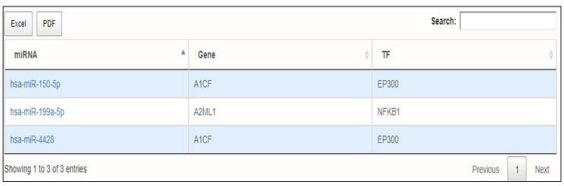

Hyperlinked 
a)

nsa-let-7c-5p

Feed Forward Loops

\section{miRNA-FFLs}

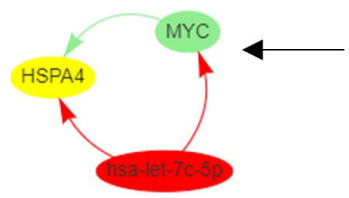

\section{TF-FFLs}

b)

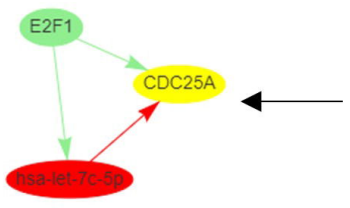

Experimentally validated targets

Excel PDF

\begin{tabular}{|l|l|l|}
\hline miRnA & Target Gene & $\begin{array}{c}\text { Target Gene (Entrez } \\
\text { Gene ID) }\end{array}$ \\
\hline hsa-let-7c-5p & TGFBR1 & 7046 \\
\hline hsa-let-7c-5p & TRIM71 & 131405 \\
\hline hsa-let-7c-5p & TRIM71 & 131405 \\
\hline hsa-let-7c-5p & TRIM71 & 131405 \\
\hline hsa-let-7c-5p & DICER1 & 23405 \\
\hline
\end{tabular}

Showing 1 to 5 of 1,090 entries
miRNA-FFLS

\begin{tabular}{|c|c|c|c|}
\hline Excel & PDF & Search: & \\
\hline \multicolumn{2}{|c|}{ miRNA } & TF & gene \\
\hline \multicolumn{2}{|c|}{ hsa-let-7c-5p } & MYC & HSPA4 \\
\hline \multicolumn{2}{|c|}{ hsa-let-7c-5p } & MYC & LDHA \\
\hline \multicolumn{2}{|c|}{ hsa-let-7c-5p } & MYC & PMAIP1 \\
\hline \multicolumn{2}{|c|}{ hsa-let-7c-5p } & MYC & TNFRSF10B \\
\hline \multicolumn{2}{|c|}{ hsa-let-7c-5p } & MYC & DDX 18 \\
\hline
\end{tabular}

\begin{tabular}{llllll}
\hline Previous & 1 & 2 & 3 & 4 & 5
\end{tabular}

\section{TF-FFLs}

\begin{tabular}{|l|l|l|l|}
\hline Excel & PDF & search: & \\
\hline miRnA & TF & gene \\
\hline hsa-let-7c-5p & E2F1 & CDC25A \\
\hline hsa-let-7c-5p & MYC & CDC25A \\
\hline hsa-let-7c-5p & SP1 & TGFBR1 \\
\hline hsa-let-7c-5p & GATA1 & DICER1 \\
\hline hsa-let-7c-5p & CEBPB & BCL2L1 \\
\hline
\end{tabular}

Showing 1 to 5 of 134 entries
Showing 1 to 5 of 144 entries 